\title{
An Improvement of Remote Control Panel for Numerous Electrical Devices in Smart Homes Using RF and Wifi
}

\author{
https://doi.org/10.3991/ijoe.v15i15.11668 \\ Trung Dang Ngoc ${ }^{(凶)}$, Linh Le Thi Huyen \\ Thai Nguyen University of Technology, Thai Nguyen, Vietnam \\ trungcsktd@tnut.edu.vn
}

\begin{abstract}
This study aims to improve a remote control panel for numerous electrical devices in smart homes using radio frequency (RF) and wifi. In this study, RF315MHz transceiver module and Arduino Ethernet Shield wifi module are combined by a programmed algorithm to transmit control signals from a central processor to the electrical devices in the smart house. The RF315 MHz transceiver with hard coding is modified to be a soft coding module in which each control channel can switch on/off 256 electrical devices. Then, a remote control panel is developed to control numerous electrical devices based on the requirements of the number of control buttons and the technology in the smart house. Moreover, this proposed method allow to control the electrical devices in the house by various panels. An experimental model with two separate transmission channels using two soft-coded RF $315 \mathrm{MHz}$ modules has been done to verify the applicability of the proposed method.
\end{abstract}

Keywords - Remote control panel, smart home, RF transmitter, RF transceiver, wifi.

\section{Introduction}

Nowadays, with a quick development of information technology and electronic engineering, smart electrical devices have been become more and more popular in smart homes. These electrical devices are commonly controlled by the remote control panels in which the control signals from the central processor to the smart devices can be transmitted by many channels such as Bluetooth waves, RF waves, wifi, Zigbee, etc. $[1,2]$. Since each control signal line has different frequencies and transmission distances, the remote control panel should be designed to adapt the requirements of each specific smart home [4, 11].

Currently, RF waves are suitable choices for smart houses because these waves provide a good signal penetration with no operating fee and the signal transmission distance is up to one kilometer. Among RF waves, RF315MHz or RF433MHz frequency bands are commonly used in transmission components and devices by many electronics companies in the world $[3,4]$. By using the same frequency band, the electric products of the different brands can be used in one application. That brings 
convenience to users when choosing or replacing any electrical device at a low cost $[3,5]$.

However, the remote control panels with the same RF waves of different electronics brands have been designed with hard coding for the transceiver and the integrated control buttons in each panel are limited $[6,9]$. Therefore, when the number of electrical devices and users increase, it may require a lot of remote control devices. That means that the number of RF transmitter modules must be increased together with inconvenience and high expenditure for users. In order to increase the flexibility of remote control panels and reduce the number of RF transmitter modules, this paper has developed a method that integrates the RF transceiver module with the Arduino Ethernet Shield wifi module [7, 12]. This solution allows the members in the smart house to control electric devices at the same time by many control devices such as smart phone, personal computer, tablet, laptop, HMI, etc [8, 10]. Since each original $\mathrm{RF}$ transceiver module is coded with 8-bit hard coding by the manufacturers, the number of communication codes are limited. To improve this problem, the hard coding RF transceiver has been transferred to the soft coding module by Arduino Uno controller card. Thus, the number of communication codes between the transmitter module and the receiver module can be increased up to $2^{8}(256)$ sets of codes. That means that each transmitter module can send up to 256 communication codes for 256 receiver modules. In other word, if one control panel has $n$ push buttons, the number of controllable devices is $256 * n$.

In order to verify the proposed method, an experimental module with two separate transmission channels using two soft RF315MHz encoders has been designed for testing.

\section{Structure of Remote Control Panel}

The proposed structure of the remote control panel is presented in Fig. 1 in which the RF communication is implemented by via RF315MHz - FS1000A transmitter module and RF315MHz - IC PT2272 receiver module [3].

The communication code on Din root of the RF315MHz transmitter module that is received the soft coding from the Arduino Uno is continuously sent to the receiver modules. Depending on the received code on each receiver module via RF waves, the relays on the corresponding electric device switch on or off.

The code of each button that is created on the monitoring interface of App inventor on the control panel is sent to the Arduino Uno via wifi of Arduino Ethernet Shield. This code is processed to be an 8-bit code, then it is combined with the series of codes of the RF transmitter module. Therefore, the RF transmitter module received the series of 24-bit datum from Arduino Uno and combined with the local wifi network from the Ethernet Shield module can expand the remote control panels not only hard control panels but also many peripheral devices such as smart phone, personal computer, tablet, laptop, HMI, etc. Since each receiver module using IC2272 controls 4 devices, if the number of electrical devices to be controlled is $m$, the number of RF receiver modules to be used is $m / 4$. 
By using this proposed method, users can easily and conveniently control many electrical devices in the smart home by various peripheral devices connected via the local wifi network.

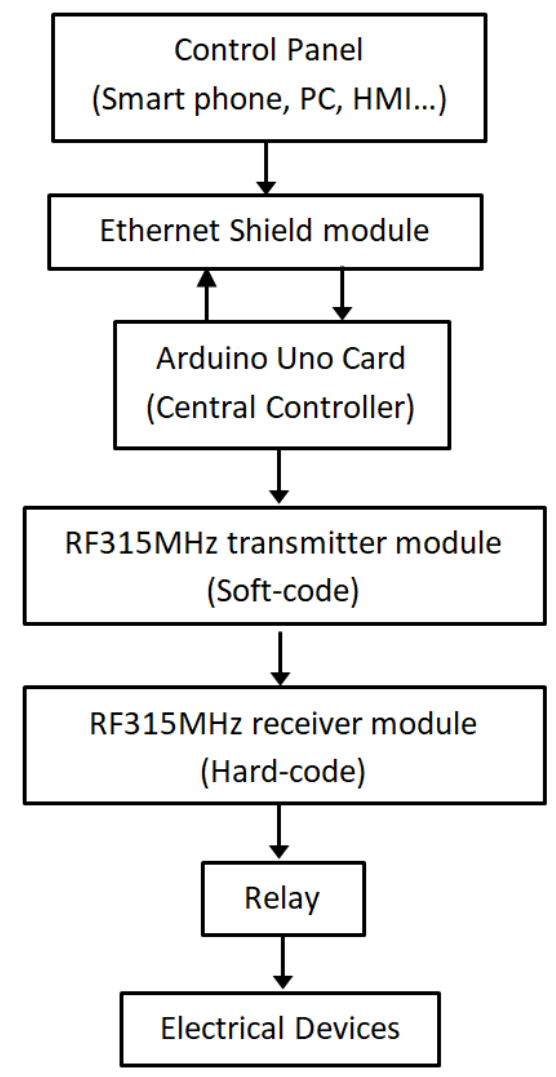

Fig. 1. The proposed control structure of remote control panel using RF $315 \mathrm{MHz}$ modules and wifi Ethernet Shield modules

\section{The Experimental Model}

In order to evaluate the applicability of this approach, the real model has been designed for testing. The developed remote control panel consists of one soft coding RF315MHz-FS1000A transmitter module and two hard coding receiver modules using IC 2272. The $\mathrm{C}++$ programming language is used for Arduino Uno card to generate soft coding communication between the transmitter module and the corresponding receiver modules. In addition, using the control interface with buttons designed by App Inventor software, the code of each button is transmitted via wifi wave to the Arduino Uno card for processing. 
The experimental model has used 2 transmission channels including RF315 module and wifi Shield Shield module to control switching on and off 4 lights as shown in Fig. 2. The connection diagrams of the system components including Arduino Uno card, Ethernet Shield module, RF transmitter module, RF315MHz receiver module and relays are presented from Fig. 3 to Fig. 5. The control panel interface with the control buttons is designed on the App Inventor on the peripheral devices as shown in Fig. 6.

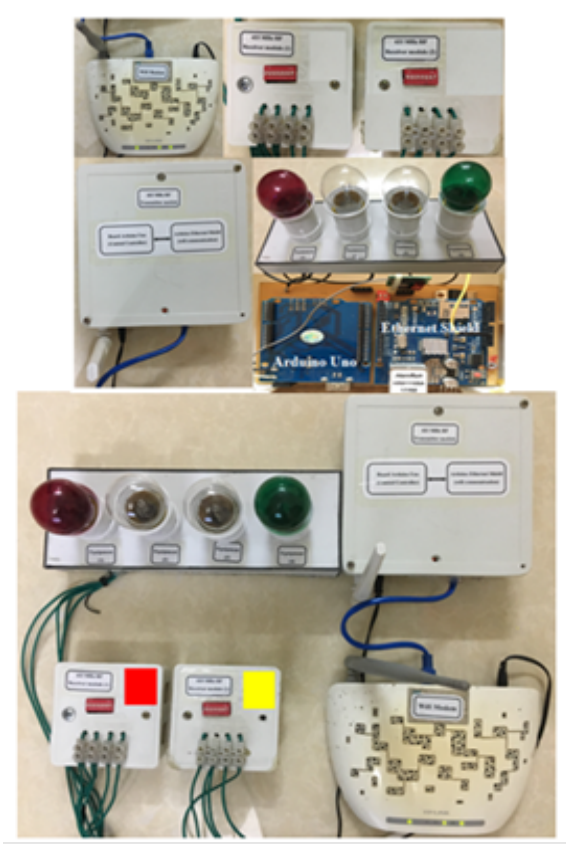

Fig. 2. The experimental model using two channels including RF315 module and wifi Shield Shield module

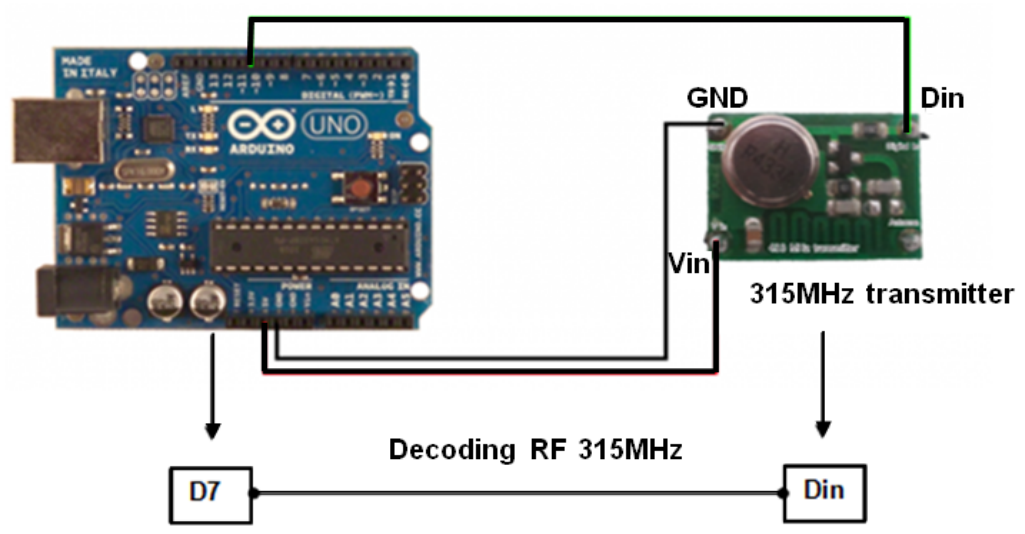

Fig. 3. The connecting diagram between Arduino Uno card with RF transmitter module 


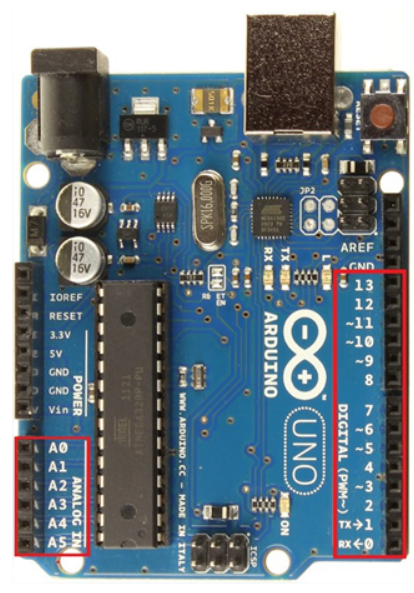

Board Arduino UNO

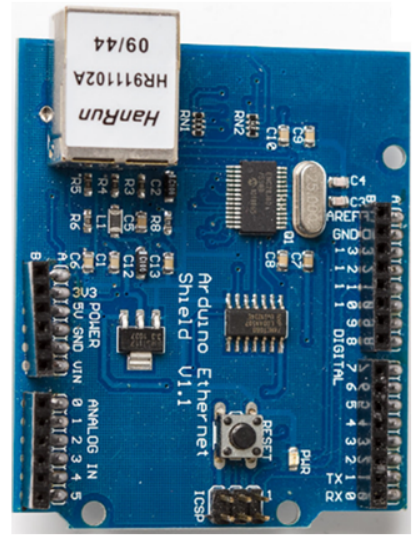

Board Ethernet Shield

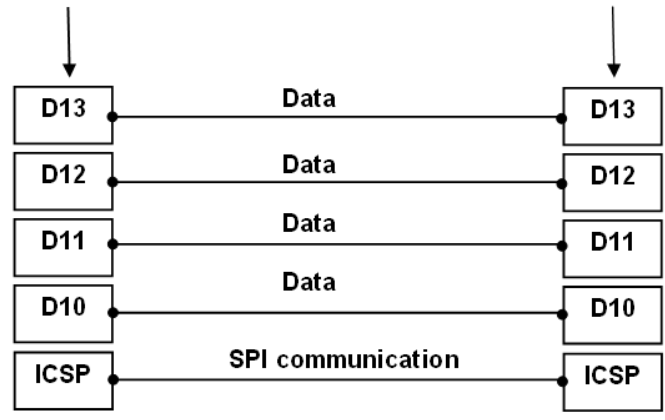

Fig. 4. Diagram of data communication between Arduino Uno card and Ethernet Shield module

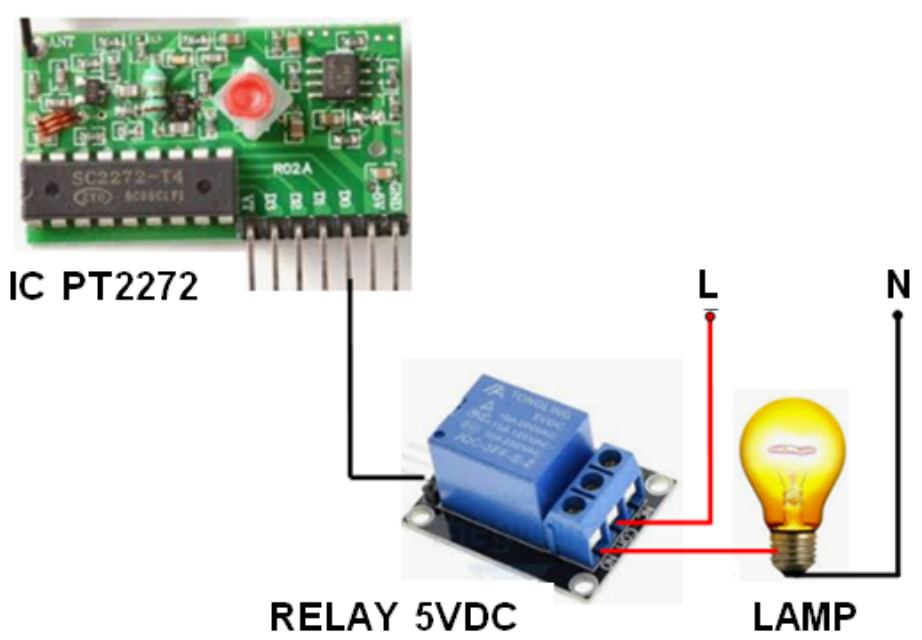

Fig. 5. The connecting diagram between RF315MHz receiver module with relays 


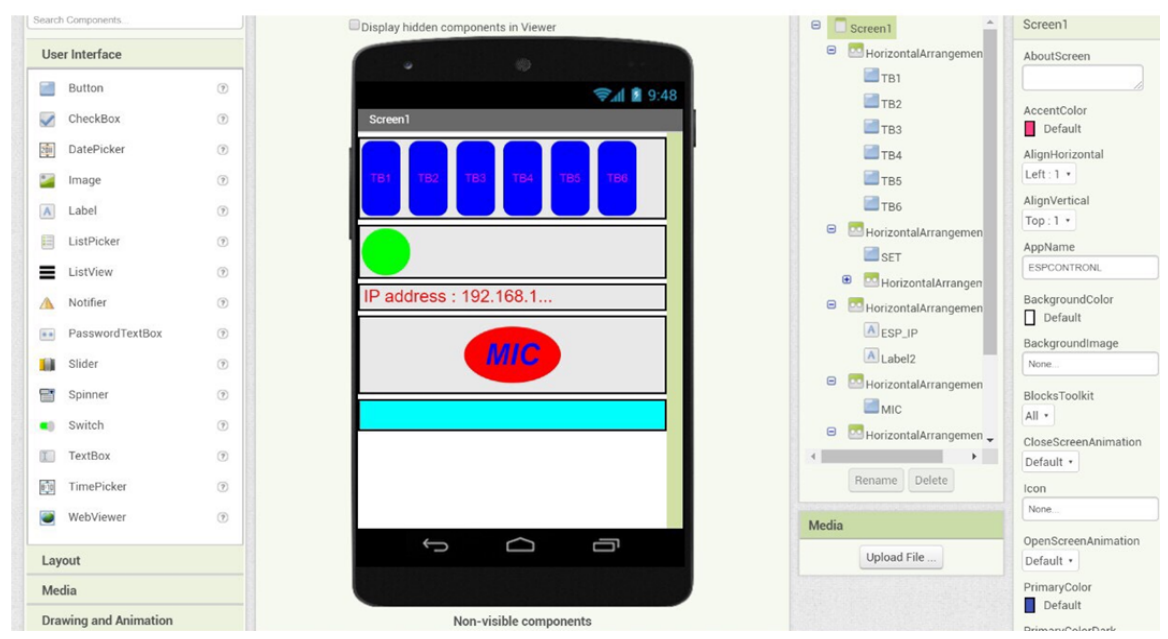

Fig. 6. Control panel interface on the App Inventor

\section{Results and Discussion}

After testing the experimental model, the control signal is obtained good quality and high accuracy. The use of the control buttons and the IP address of the local wifi designed on the App Inventor interface allows users to expand the number of the controlled electrical devices via Ethernet Shield module. In addition, as mentioned above, by using the RF315MHz receiver module with 4 addresses for each module, the maximum number of the controlled devices for this model is up to $4 \times 2^{8}$ or 1024 devices.

\section{Conclusion}

The paper has developed a method to improve a remote control panel for various electrical devices in smart homes using RF and wifi. The integration of RF transceiver module with the Arduino Ethernet Shield wifi module as well as the coded buttons on the control panel using the App Inventor allows members in the smart house to easily and conveniently control many electrical devices by various peripheral devices such as smart phone, personal computer, tablet, laptop, HMI, etc. The use of RF transceiver module with 8-bit soft coding generated by Arduino Uno controller card increases number of the controlled electrical devices. This method not only reduces the expenditure but also increases the flexibility for the control system. The good quality and high accuracy of the control signal have been achieved in the experimental model. Thus, this proposed method can be applied for the remote control systems in smart houses in reality. 


\section{$6 \quad$ References}

[1] Shantanu K. Nath, Sayera Aznabi, Nabila T. Islam, Annan Faridi, Wayesh Qarony (2017). Investigation and Performance Analysis of Some Implemented Features of the ZigBee Protocol and IEEE 802.15.4 Mac Specification: iJOE - Vol. 13, No. 1, https://doi.org/10.39 91/ijoe.v13i01.5984

[2] Hilary I. Okagbue, Muminu O. Adamu, Timothy A. Anake (2019). Differential Evolution in Wireless Communications -A Review: iJOE - Vol. 15, No. 11, pp 29-52 https://doi.org/ 10.3991/ijoe.v15i11.10651

[3] Michael P. Gaynor (2007), System-in-Package RF Design and Applications, Artech House, Inc.

[4] Juan Carlos Augusto, Chris D. Nugent (2006). Designing Smart Homes. Springer-Verlag Berlin Heidelberg

[5] Qing Wan, Ming-Jiang Weng, Song Liu (2019). Optimization of Wireless Sensor Networks based on Differential Evolution Algorithm: iJOE - Vol. 15, No. 1, https://doi.org/ 10.3991/ijoe.v15i01.9786

[6] Cheng Huang, Yongbo Lv. (2018). An Internet of Things System Based on Device-toDevice Communication Technology and Radio-Frequency Identification: iJOE - Vol. 14, No. 10, pp 210-218. https://doi.org/10.3991/ijoe.v14i10.9302

[7] V. M. Cvjetkovic, U. Stankovic (2017). Arduino Based Physics and Engineering Remote Laboratory: iJOE - Vol. 13, No. 1, pp 87-105. https://doi.org/10.3991/ijoe.v13i01.6375

[8] Hazim Shakhatreh (2019). Optimal Placement of a UAV to Prolong the Lifetime of Indoor Wireless Devices: Universal Journal of Electrical and Electronic Engineering 6(3): 108114. https://doi.org/10.13189/ujeee.2019.060304

[9] Chunmei Liu, Bin Li, Guohong Shi (2017). Design of Electronic Remote Control System Based on Wireless Sensor: iJOE - Vol. 13, No. 5, pp 146-159 https://doi.org/10.3991/ ijoe.v13i05.7057

[10] Fangzhou He (2019). Exploration of Distributed Image Compression and Transmission Algorithms for Wireless Sensor Networks: iJOE - Vol. 15, No. 1, https://doi.org/10.3991/ ijoe.v15i01.9782

[11] Jordán Pascual Espada (2014). Mobile Web-Based System for Remote-Controlled Eletronic Devices and Smart Objects: Mobile Networks and Applications, Volume 19, Issue 3, pp 435-447, https://doi.org/10.1007/s11036-014-0510-2

[12] Yang Wang (2017). Design and Implementation of a Wireless Sensor Network Node Based on Arduino: iJOE-Vol. 13, No. 11, pp 128-135, https://doi.org/10.3991/ijoe.v13i11. 7749

\section{$7 \quad$ Authors}

Trung Dang Ngoc is working in Electrical Engineering Department, Thai Nguyen University of Technology, Thai Nguyen, Vietnam.(E mail: trungcsktd@,tnut.edu.vn)

Linh Le Thi Huyen is working in Electrical Engineering Department, Thai Nguyen University of Technology, Thai Nguyen, Vietnam.

Article submitted 2019-09-12. Resubmitted 2019-10-07. Final acceptance 2019-10-07. Final version published as submitted by the authors. 